\title{
Patient Experience as a Clinical Care Component
}

\author{
Rishbha Dua ${ }^{1 *}$ and Sima Marzban ${ }^{2,3}$ \\ ${ }^{1}$ Research Fellow at Department of Research and Academic Affairs, Larkin Health System and Community Hospital, US \\ ${ }^{2}$ Vice President for Research and Academic Affairs, Larkin Health System and Community Hospital, US \\ ${ }^{3}$ Faculty, University of North Carolina, Gillings School of Global Public Health, SPH Academic Affairs, US
}

*Corresponding author: Rishbha Dua, Research Fellow at Department of Research and Academic Affairs, Larkin Health System and Community Hospital, Miami, Fl, US

\begin{abstract}
A range of values and strategies like patient satisfaction, patient-centeredness, and patient experience entered the health trajectory over time. Still, a significant percentage of medical professionals believe in a paternalistic approach associated with care delivery and follow-ups due to an asymmetry of information between clinical teams and care recipients. By providing insight into how patient satisfaction impacts clinical care components and embracing the notion of long-term improvement of healthcare, this article contributes to a better understanding of how medical practices can be improved. We aim to propose various solutions, to decrease the gap between physicians' awareness and cultivate their daily clinical care practices.
\end{abstract}

\begin{abstract}
Main Text
Translation of customer-centered concepts from other industries to healthcare has evolved the healthcare providers' understanding of patient views and experiences. A range of values and strategies like patient satisfaction, patient-centeredness, and patient experience entered the health trajectory over time. Still, a remarkable percentage of medical professionals believe in a paternalistic approach associated with care delivery and followups due to an asymmetry of information between clinical teams and care recipients. Those beliefs are rooted in having minimal insights on how patient satisfaction, interaction, and experience can affect their practice at large, and some physicians may even find it a boreoarctic component of care administration.
\end{abstract}

While most industries recognize customer satisfaction as a tool to improve their primary business outcomes, a part of the healthcare industry views it as a non-clinical administrative measure required for reimbursement and documentation purposes [1]. It is possible to change this mindset by understanding the positive impact patient experiential feedbacks and perspectives have on clinical services and healthcare outcomes. Providing high-quality healthcare, achieving expected results, and retaining patients as partners in their care planning and decision-making are all affected by the insights clinicians learn from patient experiences. In addition to potential impacts on practice, patients' feedback may result in ultimate results like sustained loyalty or, on the opposite hand, lead to a leak in the patient network or malpractice claims towards their practice [2]. Thus, it is a very reliable indicator in determining how successful doctors and hospitals are. Patients' level of happiness subjectively measures their satisfaction with healthcare. Although "patient satisfaction" and "patient experience" are sometimes used interchangeably, they refer to a different concept. During an encounter, the patient experience transpires according to what should have happened, whereas patient satisfaction is whether the patient's expectations are met [3].

It is essential to overcome traditional healthcare barriers like the abundance of information available on the internet where patients may compare surveys and reviews from other patients to set a higher standard for their care. Furthermore, there are substantial economic ramifications of patient satisfaction affecting a healthcare practice. According to statistics, the loss of a patient due to dissatisfaction can cost an average practice more than $\$ 200,000$ in lost income during their practice lifetime [4]. Additionally, a study conducted in Voluntary Hospitals of America found that nearly 70\% of patients would pay more money to consult a qualified physician of their choice [5]. Hence, physicians and the healthcare industry must evaluate patient perspectives as a professional component of care rather than a market-saving activity to succeed. Literature 
indicates that health care delivery needs to be reorganized to meet the needs of patients. Several non-medical factors contribute to patient satisfaction, including the quality of clinical services provided, the availability of medicine, the behavior of physicians and other health staff, the cost of services, hospital infrastructure, physical comfort, emotional support, and respect for individual preferences [6].

An important step is by providing excellence to your patients: Remember that each patient's journey is unique according to their expectations and based on their age, financial standing, and personal circumstances. For instance, an older person may be reliant on public transportation, while a mother of three may need assistance to get her children to their appointment. Providing patients with the option to schedule their consultation conveniently, having a pleasant medical staff to greet and treat them, and providing telephone services to handle high-stress patients are ways to address this problem. The solution is to narrow the gap between physicians' understanding and their daily clinical care practice by increasing awareness of patient views and reflections about their pain points and reliefs from all aspects of the service over the care journey. Incorporating patient and family insights into medical education, Continued Medical Education (CME), quality improvement activities, scholarly articles, and informational podcasts dedicated to bringing patient experiential knowledge into practice planning will provide physicians with the framework to embed patient experience and reported outcomes in their daily practices. Hospital Consumer Assessment of Healthcare Providers and Systems survey is the first national, standardized, publicly reported survey of patient's perspectives of hospital care [7]. We could implement a similar standard format for patient experience surveys that may help individual practices to improve the quality of care provided and further reap the benefits of meeting patient expectations.

\section{References}

1. White B (1999) Measuring Patient Satisfaction: How to Do It and Why to Bother. FPM.

2. Prakash B (2010) Patient satisfaction. Journal of cutaneous and aesthetic surgery 3(3): 151-155.

3. Eisenberg A MD (2000) what is patient satisfaction and why does it matter? Health.

4. Luecke RW, Rosselli VR, Moss JM (1991) The economic ramifications of "client" dissatisfaction. Group Pract J 40: 8-18.

5. Sodani PR, Kumar RK, Srivastava J, Sharma L (2010) Measuring patient satisfaction: a case study to improve quality of care at public health facilities. Indian journal of community medicine 35(1): 52-56.

6. Voluntary Hospitals of America (1988) Special Report: Quality Care. Market Monitor 11.

7. (2021) HCAHPS: Patients' Perspective of Care Survey/ CMS. CMS gov.

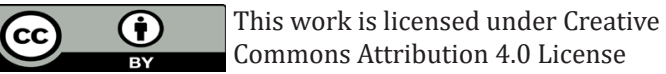

To Submit Your Article Click Here: Submit Article

DOI: 10.32474/RRHOAJ.2021.07.000251

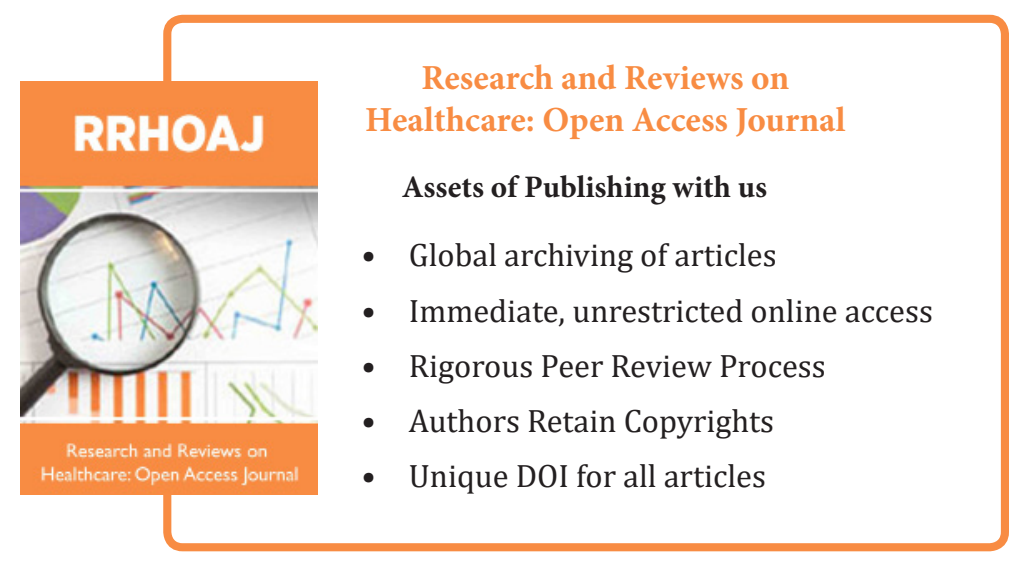

